\title{
ENSAMBLES DE AVES EN EL DESIERTO DE ATACAMA, NORTE GRANDE DE CHILE
}

\section{AVIAN ASSEMBLAGES OF ATACAMA DESERT, FAR NORTH OF CHILE}

\author{
Alberto Gantz, Jaime Rau \& Enrique Couve \\ Laboratorio de Ecología. Departamento de Ciencias Básicas. Universidad de Los Lagos, Casilla 933. Osorno, Chile. \\ E-mail: agantz@ulagos.cl
}

\section{RESUMEN}

Se evaluó la riqueza y composición de especies de aves en el desierto de Atacama, Norte Grande de Chile, entre 1996 y 1998. Mediante transectos lineales se estimó la riqueza de especies de aves en 9 localidades del desierto de Atacama agrupadas en cuatro zonas ecológicas: Desierto Litoral, Desierto Interior, Tropical Marginal y Tropical de Altura. La riqueza total de aves fue de 80 especies distribuidas en 51 géneros y 26 familias. La riqueza total observada corresponde al 52\% de las aves históricamente descritas para el desierto del Norte Grande de Chile. Las zonas ecológicas del desierto litoral y tropical de altura poseen elementos avifaunísticos únicos para cada zona. Las aves insectívoras predominaron entre los demás gremios tróficos ( $40 \%$ del total de especies). La predominancia de los diferentes gremios tróficos parece estar determinada por el tipo de hábitat utilizado para alimentarse. Nuestros resultados permitirían sugerir que la riqueza de especies de aves en el desierto de Atacama, Norte Grande de Chile, estaría d eterminada por la heterogeneidad del hábitat e inmigración de especies provenientes de zonas adyacentes al desierto de Atacama.

Palabras Clave: Aves terrestres, Chile, desierto, diversidad.

\section{ABSTRACT}

The richness and composition of bird species in the Atacama desert located in the Far North of Chile, was studied between 1996 and 1998. Using lineal transects, the richness of bird species was evaluated in nine locations of the Atacama Desert, grouped into four ecological zones: Coastal Desert, Inland Desert, Marginal Tropical, and Altitude Tropical. Bird species richness totaled 80 species, belonging to 51 genera and 26 families. Total richness corresponded to $52 \%$ of historical descriptions of birdlife in the Chilean Far North desert. The ecological zones of the coastal and altitude tropical desert areas each have unique bird fauna elements. Insectivorous birds predominated over other trophic groups ( $40 \%$ of total species). The predominance of different trophic groups appears to depend on the type of feeding habitat used. Our results suggest that the variety of bird species in the Chilean Atacama Desert is determined by habitat heterogeneity and the immigration of species from zones, adjacent to the Atacama Desert.

KEYwords: Terrestrial birds, desert, diversity, Chile. 


\section{INTRODUCCION}

Los patrones de riqueza y diversidad de especies están determinados por factores ambientales y biológicos, que actuarían simultáneamente sobre los ensambles vegetacionales y faunísticos tanto a nivel local como regional (Ricklefs 1987). A nivel local, la riqueza de especies estaría determinada por diversos factores y procesos que estructurarían las comunidades ecológicas, tales como depredación, exclusión competitiva y tasas de perturbación (Cornell \& Karlson 1996; Morton 1993; Ricklefs 1987); a escala regional participarían procesos histórico-evolutivos tales como especiación, endemismo y dispersión geográfica (Cornell 1985,1993, Morton 1993, Schlutter \& Ricklefs 1993).

La riqueza de especies local y regional muestra una influencia mutua, dependiendo si corresponden a comunidades interactivas o no interactivas (sensu Cornell 1993). En las comunidades interactivas, la riqueza local de especies está determinada por interacciones bióticas que imponen un límite al número local de especies, independiente de la riqueza regional. Cuando esto ocurre, las comunidades se consideran "saturadas" (sensu Cornell 1993; Ricklefs 1987). Por el contrario, en las comunidades no interactivas, la riqueza local de especies incrementa proporcionalmente con la riqueza regional, muestra una dependencia lineal y las comunidades se consideran "no saturadas" (Caley \& Schlutter 1997; Cornell \& Lawton 1992).

Los ecosistemas áridos constituyen sistemas ecológicos relativamente simples, con baja productividad y riqueza de especies (Noy-Meyer 1973), pero con una alta heterogeneidad espacial (Tongway et al. 2004, Kelt et al. 1996). Esta característica favorecería el desarrollo de estudios sobre los procesos estructuradores de las comunidades ecológicas y los patrones de diversidad de especies (Kelt et al. 1996; Wiens 1991).

Por otra parte, en las zonas áridas, la cobertura y heterogeneidad vegetacional y la disponibilidad de agua utilizable por las aves constituyen factores causales que determinan la organización de las comunidades ecológicas (Blendinger 2005) y establecen un patrón espacial de recursos disponibles para los consumidores (Wiens 1985, Rosenzweig 1995).

En las zonas áridas del Norte Grande de Chile, los estudios sobre los procesos y patrones que determinan la diversidad de especies están centrados fundamentalmente sobre micromamíferos (Kelt et al. 1996; Marquet 1994; Meserve \& Glanz 1978), pero véanse Spotorno et al. (1998), Rau et al. (1998) y Jerez (2000) para estudios actualizados sobre diversidad, adaptación y distribución geográfica de mamíferos, aves e insectos fitófagos en esta zona geográfica. No obstante, los estudios sobre los ensambles de aves en ecosistemas áridos de Sudamérica son relativamente escasos (Blendinger 2005).

Como parte de un estudio más extenso sobre biodiversidad, clima y evolución adaptativa de vertebrados de las zonas áridas del norte de Chile, en el presente estudio presentamos antecedentes sobre la riqueza y composición de los ensambles de aves terrestres presentes en ecosistemas áridos de la Provincia de Antofagasta, Norte Grande de Chile.

\section{MATERIALES Y METODOS}

\section{ÁRea DE ESTUdio}

Durante el invierno 1996, otoño e invierno de 1997 y el verano de 1998 se muestrearon nueve localidades en el desierto de Atacama, II Región Administrativa de Chile. Las nueve localidades se agruparon en cuatro zonas ecológicas (desierto litoral, desierto interior, tropical marginal y tropical de altura), cuyas comunidades vegetales difieren estructural y florísticamente (Gajardo 1994).

La zona del desierto litoral está dominada florísticamente por Distichlis spicata (L.) Green., Tessaria absinthioides (Hook. \& Arn.) y Atriplex spp. (Philippi). En el desierto interior dominan los cultivos intensivos de trigo (Triticum aestivum (L.), Fam. Poaceae), maíz (Zea mays (L.), Fam. Poaceae) y alfalfa (Medicago sativa (L.), Fam. Fabaceae), entre otros (IGM 1983); árboles y arbustos frutales y ornamentales como: Higueras (Ficus carica (L.), Fam. Moraceae), membrillos (Cydonia oblonga (Miller), Fam. Rosaceae), granados (Punica granatum (L.), Fam. Punicaceae) y álamos (Populus nigra var. Italia (Du Roi), Fam. Salicaceae). La zona tropical marginal constituye una formación vegetacional altiplánica representada por Calamagrostis rigescens ((Presl.) Scribn. Fam. Graminaceae) y Oxicloe andina ((Philippi) Fam. Juncaceae) y la zona tropical de altura (sobre los $3.000 \mathrm{~m}$ s.n.m.) está constituida por gramíneas xerófitas y perennes de la familia Poaceae como: 
Stipa sp.(Phil.?), Festuca sp. (Presl.) Kunth. y Distichlis spp.(L.) Green. y hierbas perennes (Fuenzalida 1965) (para una descripción más detallada de las comunidades vegetacionales y características climáticas del desierto de Atacama, véase Gajardo 1994; Fjeldsa \& Krabbe 1990; Instituto Geográfico Militar 1983 y Fuenzalida 1965).

\section{Censos de aves}

Durante el período de estudio se realizaron censos de aves terrestres en las cuatro zonas ecológicas seleccionadas. La riqueza de especies de aves y sus abundancias relativas se evaluó con el método del transecto de ancho indefinido (Bibby et al. 1992). Este método se consideró adecuado dado el carácter lineal y ancho variable de los hábitats estudiados (Bibby et al. 1992). Se contabilizaron todas las aves vistas y oídas. Todas las aves fueron observadas con ayuda de binoculares e identificadas, de ser necesario, con ayuda de la guía de las aves de Chile de Araya \& Millie (1986). La longitud de los transectos fue de aprox. $1.500 \mathrm{~m}$, se recorrieron unidireccionalmente y se realizaron durante una hora. Todos lo censos se efectuaron durante la mañana, entre las 8:00 y 11:00 h. En cada localidad los transectos se recorrieron una vez al día por dos días.

ANÁLISIS DE LOS ENSAMBLES DE AVES

Se evaluó la variación temporal y espacial de los ensambles de aves en las cuatro zonas ecológicas áridas del norte de Chile. El análisis temporal consideró la variación de la composición y riqueza de especies de los ensambles de aves durante las cuatro excursiones realizadas entre 1996-1998. El análisis espacial se basó en un análisis de similitud de la riqueza de aves entre las cuatro zonas ecológicas evaluadas. El grado de similitud de los ensambles de aves entre las zonas ecológicas se evaluó utilizando el índice de similitud de Morisita. Este fue considerado el método más adecuado porque presenta una mayor independencia del tamaño muestral (Krebs 1989) comparativamente con índices de similitud alternativos. Este índice varía entre $0=$ mínima similitud a $1=$ máxima similitud.

Todas las especies de aves observadas fueron incorporadas en cinco categorías tróficas de acuerdo a la información sobre las dietas de las aves señaladas por diversos autores (Lopez-Callejas
1995; Marone et al. 1997; Marone 1992; Araya \& Millie 1986; Johnson 1965; Housse 1945). Las categorías tróficas seleccionadas fueron: insectívoras, granívoras, carnívoras, omnívoras y herbívoras.

ANÁLISIS DE RIQUEZA Y COMPOSICIÓN DE ESPECIES DE AVES La riqueza local de especies de aves la cuantificamos como el número de especies presentes en cada zona ecológica (diversidad $\alpha$ ) (e.g.: Morton 1993). La diversidad $\beta$ (DB), número diferencial de especies entre los hábitats (Rosenzweig 1995; Schlutter \& Ricklefs 1993), se evaluó aplicando la fórmula propuesta por Whittaker (1972): $\mathrm{DB}=(\mathrm{Sc} / \mathrm{S})-1$; donde, Sc es la riqueza de especies combinada entre dos hábitats y $\mathrm{S}$ es el número promedio de especies de las muestras $\alpha$ comparadas (ver detalles en Rau et al. 1998). Puesto que localidades únicas no poseen valores de diferenciación, al coeficiente $\mathrm{Sc} / \mathrm{S}$ se le restó la unidad (Whittaker 1972). De este modo, la DB relativa varió entre 0 (i.e., muestras idénticas) y 1 (i.e., muestras totalmente diferentes). La riqueza regional de especies de aves (diversidad $\gamma$ ), número de especies en un paisaje o región geográfica (Rosenzweig 1995), se evaluó como el número total de especies de aves observadas en la macrozona desértica estudiada.

\section{RESULTADOS}

La riqueza total de especies de aves terrestres observada en el área de estudio durante las cuatro excursiones fue de 80 especies pertenecientes a 51 géneros y 26 familias (véase listado de especies y familias de aves en el apéndice I en Spotorno et al. 1998). Las 80 especies observadas representan el $52 \%$ de aves terrestres descritas históricamente (153 especies) en las zonas áridas del Norte Grande de Chile. La diferenciación en la composición de especies entre las zonas ecológicas estuvo determinada por 21 especies en la zona tropical marginal y 57 especies para la tropical de altura, con una media (aritmética) de 38 especies en las cuatro zonas ecológicas analizadas. La riqueza local de especies de aves entre las zonas ecológicas fue intermedia $(\mathrm{BD}=0,481 \pm 0,093)$.

Los gremios tróficos predominantes fueron las aves insectívoras que representan el $40,0 \%$ del total de aves, seguidas por las granívoras que también constituyó un grupo importante (28,8\%). Las carnívoras estuvieron 
TABla I. Distribución del número de especies de aves agrupadas en cada categoría trófica, de acuerdo a las zonas ecológicas y al total de especies observadas en el desierto de Atacama, Norte Grande de Chile.

TABLE I. Distribution of the number of bird species grouped into each trophic category, according to ecological zones and total number of species observed in the Atacama desert of the Chilean Far North.

\begin{tabular}{|c|c|c|c|c|c|c|}
\hline \multirow{2}{*}{$\begin{array}{l}\text { Categorías } \\
\text { Tróficas }\end{array}$} & \multicolumn{2}{|c|}{ Especies } & \multirow{2}{*}{$\begin{array}{c}\text { Desierto } \\
\text { Litoral }\end{array}$} & \multirow{2}{*}{$\begin{array}{l}\text { Desierto } \\
\text { Interior }\end{array}$} & \multirow{2}{*}{$\begin{array}{r}\text { Tropical } \\
\text { Marginal }\end{array}$} & \multirow{2}{*}{$\begin{array}{c}\text { Tropical de } \\
\text { Altura }\end{array}$} \\
\hline & $\mathrm{N}^{\mathrm{o}}$ & $\%$ & & & & \\
\hline Insectívoras & 32 & 40,0 & 18 & 12 & 8 & 22 \\
\hline Granívoras & 23 & 28,8 & 6 & 15 & 10 & 20 \\
\hline Carnívoras & 14 & 17,5 & 11 & 3 & 1 & 8 \\
\hline Frugívoras & 2 & 2,5 & 0 & 2 & 0 & 1 \\
\hline Herbívoras & 1 & 1,2 & 0 & 0 & 1 & 0 \\
\hline Omnívoras & 6 & 7,5 & 2 & 4 & 1 & 4 \\
\hline Nectarívoras & 2 & 2,5 & 0 & 0 & 0 & 2 \\
\hline TOTAL & 80 & 100 & & & & \\
\hline
\end{tabular}

TABLA II. Matriz de similitud (Índice de Morisita) de la composición de especies de los ensambles de aves entre las 4 excursiones realizadas entre 1996 y 1998 en el desierto de Atacama, Norte Grande de Chile.

TABLE II. Similarity matrix (Morisita Index) of the species composition of bird assemblages during the 4 excursions undertaken between 1996 and 1998 in the Atacama desert of the Chilean Far North.

\begin{tabular}{ccccc}
\hline Excursiones & Invierno 1996 & Otoño 1997 & Invierno 1997 & Verano 1998 \\
\hline Invierno 1996 & ------ & 0,5499 & 0,5007 & 0,2769 \\
Otoño 1997 & ----- & ----- & 0,1169 & 0,6611 \\
Invierno 1997 & ------ & ----- & ----- & 0,0578 \\
\hline
\end{tabular}

representadas por menos del 20\%, mientras que las restantes categorías, frugívoras, herbívoras y nectarívoras, representaron menos del $10 \%$ del total de especies (Tabla I). Así mismo, los insectívoros dominaron en el desierto litoral y tropical de altura, mientras que los granívoros fueron predominantes en el desierto interior y tropical de altura (Tabla I).

El nivel de similitud de la composición de especies de los ensambles de aves entre las cuatro excursiones, como era de esperarse, mostró variaciones particularmente entre las estaciones contrastantes de invierno y verano (Tabla II).

Los insectívoros dominaron en las tres primeras excursiones, reemplazados por los granívoros en el verano de 1998. Destaca el incremento poblacional de los granívoros durante el invierno de 1997 (Tabla III), aunque éste incremento fue explicado principalmente por el arribo de poblaciones numerosas de Sicalis luteola (Meyen).

El grado de similitud de los ensambles de aves en el área estudiada del norte de Chile muestra relación con el grado de cercanía de las zonas ecológicas. Las zonas ecológicas adyacentes comparten entre sí un mayor número de especies (i.e., zona tropical marginal con la zona tropical de altura y el desierto litoral con el desierto interior) en contraste con zonas ecológicas distantes entre si (Tabla III). Esto se evidencia por la variación en el número de especies únicas presentes en cada zona ecológica (Tabla IV).

\section{DISCUSION}

La riqueza total de especies de aves terrestres residentes en el desierto de Atacama es comparativamente mayor que en otras zonas áridas del mundo. De acuerdo a Araya \& Millie (1986), 153 especies de aves terrestres estarían presentes históricamente en esta zona. En contraste Morton 
TABLA III. Matriz de similitud (Indice de Morisita) de los ensambles de aves entre las cuatro de zonas ecológicas en los ecosistemas áridos de Atacama, Norte Grande de Chile, entre 1996-1998.

TABLE III. Similarity matrix (Morisita Index) of bird assemblages in four ecological zones in the arid ecosystems of the Atacama desert of the Chilean Far North, between 1996 and 1998.

\begin{tabular}{ccccc}
\hline Tipo de Hábitat & Desierto Litoral & Desierto Interior & Tropical Marginal & Tropical de Altura \\
\hline Desierto Litoral & ----- & 0,5667 & 0,0062 & 0,0093 \\
Desierto Interior & ----- & ----- & 0,3051 & 0,3338 \\
Tropical Marginal & ------ & ----- & ------ & 0,8024 \\
\hline
\end{tabular}

TABLA IV. Número de especies exclusivas para cada zona ecológica estudiada entre 1996 y 1998 en al desierto de Atacama, Norte Grande de Chile. (I) = especie exótica introducida.

TABLE IV. Number of species exclusive to each ecological zone studied between 1996 and 1998 in the Atacama desert, of the Chilean Big North. (I) = introduced exotic species.

\begin{tabular}{|c|c|c|}
\hline Tipo de Hábitat & $\mathrm{N}^{\mathrm{o}}$ de especies únicas & Especies \\
\hline Desierto Litoral & 6 & $\begin{array}{l}\text { Cathartes aura, Coragyps atratus, Vultur gryphus, Pandion } \\
\text { haliaetus, Caracara plancus, Chordeiles acutipennis }\end{array}$ \\
\hline Desierto Interior & 12 & $\begin{array}{c}\text { Athene cunicularia, Glaucidium nanum, Tyto alba, Falco } \\
\text { sparverius, Tachycineta meyeni, Turdus falcklandii, Thraupis } \\
\text { bonariensis, Columba livia (I), Xolmis pyrope, Muscisaxicola c. } \\
\text { cinerea, Anthus correndera, Thynocorus orbignyianus, }\end{array}$ \\
\hline Tropical Marginal & 3 & $\begin{array}{c}\text { Pterocnemia pennata, Geositta rufipennis, Muscisaxicola } \\
\text { albifrons }\end{array}$ \\
\hline Tropical de Altura & 15 & $\begin{array}{c}\text { Mimus triurus, Geranoaetus melanoleucus, Bubo magellanicus, } \\
\text { Phegornis mitchellii, Gallinago andina, Oreotrochilus } \\
\text { leucopleurus, Oreotrochilus stella, Upucerthia ruficauda, } \\
\text { Upucerthia andaecola, Muscisaxicola frontalis, M. flavicucha, } \\
\text { Sicalis auriventris, Phrygilus unicolor, Phrygilus dorsalis, } \\
\text { Phrygilus erythronotus }\end{array}$ \\
\hline
\end{tabular}

(1993) reportó 140 especies de aves terrestres para el desierto Australiano y Wiens (1991) señaló130 especies para el desierto de Sonora en Estados Unidos.

Si bien nuestros datos son escasos para establecer conclusiones, consideramos que probablemente procesos de inmigración y colonización del área por elementos avifaunísticos del pool regional y la alteración del hábitat por influencia antrópica, constituyen dos factores que permitirían explicar la alta diversidad local de especies de aves observada. La alta proporción de especies que comparten los ecosistemas áridos con ecorregiones adyacentes sugiere que estas ecorregiones se comportarían como hábitat fuente, que aportarían elementos avifaunísticos al desierto de Atacama. La incorporación de especies desde el pool regional constituye un factor importante para mantener la diversidad local de especies (Morton 1993).

Esto se evidencia al trazar arbitrariamente una línea longitudinal imaginaria a una altitud media de 2.400 m s.n.m. que divida el desierto de Atacama en una zona alta $(>2.400 \mathrm{~m}$ s.n.m) y una baja $(<2.400 \mathrm{~m}$ s.n.m.). Se observó una marcada diferenciación de los elementos constituyentes de los ensambles de aves en ambas zonas (Morisita $=0,1818)$. Además, los ensambles avifaunísticas muestran importantes afinidades con elementos de macrorregiones cercanas. El 87\% (41 de 47 especies) de las aves de las zonas de litoral (zona baja), poseen una 
distribución geográfica más amplia que incluyen la zona semiárida y mediterránea de Chile. Las aves de las zonas tropical marginal y de altura (zona alta) comparten el 87\% (52 de 60 especies) con la zona del Monte, Argentina (los antecedentes de las aves argentinas se obtuvo de Canevari et al. 1991), el 70\% con ecosistemas mediterráneos chilenos (42 de 60 especies) y el 55\% (33 de 60 especies) con la región preandina y altiplánica de Bolivia (los datos de las aves de Bolivia se obtuvieron de Kempff 1985). Estos patrones probablemente están asociados a las similitudes vegetacionales que comparten estas ecorregiones. Al respecto, Spotorno \& Veloso (1990) señalaron una alta similitud del número de géneros de plantas entre la zona de la Puna y la Patagonia y entre el desierto de la Sierra (Perú) con Chile central. Además, la transformación antrópica de los hábitats ribereños naturales, en los cuales se concentra la mayor parte de la actividad biológica en zonas desérticas (Marquet et al. 1998), en hábitats ribereños agrícolas que ofrecen una alta disponibilidad de recursos tróficos (particularmente semillas y frutos), una alta heterogeneidad estructural del hábitat (pluriestratificados) y una importante oferta de agua dulce utilizable por las aves, podrían constituir los factores causales de la riqueza local de aves observada. Así mismo, Marquet et al. (1998) documentó que los ecosistemas ribereños pueden funcionar como corredores biológicos para la flora y la fauna, lo cual permitiría explicar la similitud de los elementos avifaunísticos entre las zonas ecológicas adyacentes observada en el presente estudio.

Así mismo, una mayor heterogeneidad de hábitat es un factor determinante de una alta diversidad $\beta$ (Whittaker 1972). Los valores intermedios de diversidad $\beta$ observados, probablemente sean consecuencia de los patrones de heterogeneidad espacial que caracterizan diversas regiones áridas (Kelt et al. 1996), a factores físicos del hábitat (Fjeldsa \& Krabbe 1990) y a requerimientos ecológicos particulares de las especies (Whittaker 1972). La segregación de las comunidades avifaunísticas, entre las zonas altas y las zonas bajas, corrobora lo anterior. El desierto absoluto (productividad primaria casi nula y aridez casi absoluta (Gajardo 1994), probablemente actúa como una barrera que limita el intercambio de algunas especies de aves entre ambas zonas, como también lo sugiere Marquet (1994) para micromamíferos.
La dominancia de los insectívoros parece ser un patrón común en los ecosistemas áridos del continente americano. Mares \& Rosenzweig (1978) reportó una dominancia de los insectívoros en el desierto del Monte, Argentina y Wiens (1991) lo señala para el desierto de Sonora, USA. La mayor riqueza de especies y variación espacial de los insectívoros probablemente constituye una respuesta ante variaciones en la abundancia y diversidad de insectos asociados a diferentes hábitats (Rau et al. 1998). En efecto, la mayor riqueza de aves insectívoras en las zonas ecológicas del Litoral y Tropical de altura, concuerda con la mayor riqueza de insectos coleópteros señalados por Jerez (2000) en un estudio realizado en las mismas zonas geográficas del presente estudio.

La riqueza de especies de aves granívoras depende directamente de la oferta de semillas de plantas anuales y de fuentes confiables y estables de granos para su mantención durante el período invernal (Blendinger \& Ojeda 2001; Marone et al.1997; Morton 1985; Morton \& Davies 1983; Mares \& Rosenzweig 1978). Así, las plantaciones de gramíneas y frutales en el desierto interior y, en particular, las vastas extensiones de gramíneas que caracterizan la estepa altoandina (Fuenzalida 1965; Gajardo 1994) ofrecen abundancia de recursos tróficos potencialmente explotables por las aves granívoras y permitirían explicar su alta representación en las ecorregiones tropical marginal y desierto interior.

Si bien nuestros datos son escasos como para obtener conclusiones, nos permitirían hipotetizar que la variación temporal entre las excursiones de los gremios tróficos de aves, en especial de los granívoros, podría estar asociada a dos factores: 1) variaciones en la oferta y disponibilidad de recursos tróficos determinada por las precipitaciones locales, eventualmente asociado al fenómeno climático ENOS que provoca incrementos irruptivos en la productividad primaria (semillas) en el norte de Chile (Rau et al. 1998, Jaksic et al. 1997, Jaksic 1998) y 2) el mismo fenómeno podría también favorecer la inmigración de especies debido a migraciones latitudinales y altitudinales desde zonas adyacentes como lo han señalado Blendinger (2005) y Marone et al. (1997) para el desierto del Monte en Argentina.

De este modo, la heterogeneidad del hábitat y la dispersión de especies desde zonas cercanas serían factores importantes que permitirían sostener la 
riqueza local de aves en los ecosistemas áridos del desierto de Atacama, Norte Grande de Chile. Esto concordaría con diversos estudios realizados en otras zonas áridas del mundo (Cornell \& Karlson 1996; Holt 1993; Cornell \& Lawton 1992; Ricklefs 1987). Nuestros datos obtenidos a escalas temporales y espaciales restringidas no permitirían explicar la riqueza de aves a escala regional en el desierto de Atacama, Norte Grande de Chile. Sin embargo, como a escala regional actúan procesos históricos y geográficos que afectan la composición de los ensambles de aves locales (Schlutter \& Ricklefs 1993), probablemente la riqueza regional observada en este estudio podría ser producto de su estrecha relación geográfica con las diversas áreas de endemismo adyacentes y con la zona de la Puna, principal centro de diversificación de aves durante el pleistoceno (Vuilleumier \& Simberloff 1980, Cracraft 1985). Al respecto, según Cornell \& Lawton (1992) una zona geográfica con condiciones que favorecen una rápida diversificación de linajes, resultará en altas riquezas regionales.

Estudios comparativos entre las zonas de diversificación faunística con los ecosistemas desérticos del norte de Chile permitirían contribuir a un mayor conocimiento sobre la alta riqueza regional de aves en los ecosistemas áridos de Atacama, Norte Grande de Chile.

\section{AGRADECIMIENTOS}

A los revisores anónimos cuyas importantes contribuciones permitieron mejorar el presente manuscrito. Trabajo financiado por el proyecto Fondecyt No 1980017, del Programa Sectorial Biomas y Climas Terrestres y Marinos del norte de Chile.

\section{BIBLIOGRAFIA}

Araya, B. \& Millie, G. 1986. Guía de campo de las aves de Chile. Editorial Universitaria. Santiago, Chile. 389 pp.

Bibby, C.J., Burgess, N.D. \& Hill, D.A. 1992. Bird census techniques. Academic Press. United Kingdom. $257 \mathrm{pp}$.

BLENDINGER, P.G. 2005. Abundance and diversity of smallbird assemblages in the Monte desert, Argentina. Journal of Arid Environment 61:567-587.

Blendinger, P.G. \& OJeDA, RA. 2001. Seed supply as a limiting factor for granivorous bird assemblages in the Monte Desert, Argentina. Austral Ecology 26:413-422.

Caley, M.J. \& Schlutter, D. 1997. The relationship between local and regional diversity. Ecology 78:70-80.

Canevari, M., P. Canevari, G.R. Carrizo, G. Harris, J.R. Mata \& Straneck, R.J. 1991. Nueva guía de las aves argentinas. Tomo 1. Fundacion Acindar. Buenos Aires, Argentina. 411 pp.

Cornell, H.V. 1985. Local and regional richness of cynipine gall wasps on california oaks. Ecology 66:1247-1260.

Cornell, H.V. 1993. Unsaturated patterns in species assemblages: The role of regional processes in setting local species richness. En: Species Diversity in ecological communities (R.E. Ricklefs \& D. Schlutter), pp. 243-252. The University of Chicago Press.

Cornell, H.V. \& Karlson, R.H. 1996. Species richness of reef-building corals determined by local and regional processes. Journal of Animal Ecology 65:233-241.

Cornell, H.V. \& Lawton, J.H. 1992. Species interactions, local and regional processes, and limits to the richness of ecological communities: a theoretical perspective. Journal of Animal Ecology 61:112.

CraCraft, J. 1985. Historical biogeography and pattern of differentiation within the south American avifauna: areas of endemism. American Ornithological Union, Ornithological Monographs 36:49-84.

Fjeldsa, J. \& KrabBe, N. 1990. Birds of the high Andes. Zoological Museum, University of Copenhagen and Apollo Books, Svendborg. Denmark. 876 pp.

Fuenzalida, H. 1965. Biogeografía. En: Geografía económica de Chile (Eds. CORFO), pp. 245-266. Editorial Universitaria S.A. Santiago, Chile.

Gajardo, R. 1994. La vegetación natural de Chile. Clasificación y distribución geográfica. Editorial Universitaria. Santiago, Chile. 165 pp.

Holt, R.D. 1993. Ecology at the mesoscale: The influence of regional processes on local communities. En: Species diversity in ecological communities (Eds. R.E. Ricklefs \& D. Schlutter), pp. 77-88. The University of Chicago Press.

Housse, R. 1945. Las aves de Chile y su clasificación moderna. Su vida y sus costumbres. De. Universidad de Chile. Santiago, Chile. 390 pp.

Instituto Geográfico Militar. 1983. Geografía de Chile. Biogeografía. Tomo III. Editorial Quintanilla. Santiago, Chile. 230 pp.

JAKSIC, F.M. 1998. Las múltiples facetas del fenómeno El Niño/Oscilación del Sur. Ambiente y Desarrollo 14:53-61.

Jaksic, F.M., Meserve, P.L. \& Gutierres, J.R. 1997. A long-term study of vertebrate predator response to an El Niño (ENSO) disturbance in western South America. Oikos 78:341-354.

JEREZ, V. 2000. Diversidad y patrones de distribución 
geográfica de insectos coleópteros en ecosistemas desérticos de la Región de Antofagasta, Chile. Revista Chilena de Historia Natural 73:1-17.

Johnson, A.W. 1965. The bird of Chile and adjacent regions of Argentina, Bolivia y Perú. Vol. 1 y 2. De. Buenos Aires, Buenos Aires Argentina. 398 pp.

Kelt, D., Brown, J.H., Heske, E.J., Marquet, P.A., Morton, S.R., Reid, J.R., Rogovin, K.A. \& Shenkrot, G. 1996. Community structure of desert small mammals: comparison across four continents. Ecology 77:746-761.

KempfF, N. 1985. Aves de Bolivia. Ed. Gisbert. La Paz, Bolivia. $156 \mathrm{pp}$

Krebs, Ch.J. 1989. Ecological methodology. Harper Collins Publishers. New York, USA. 654 pp.

López-Callejas, M.V. 1995. Dieta de Zonotrichia capensis (Emberizidae) y Diuca diuca (Fingillidae): efecto de la variación estacional de los recursos tróficos y la riqueza de aves granívoras en Chile central. Revista Chilena de Historia Natural 68:321-331.

Mares, M.A. \& Rosenzweig, M.L. 1978. Granivory in north and south american deserts: rodents, birds, and ants. Ecology 59(2):235-241.

Marone, L. 1992. Seasonal and year-to-year fluctuations of bird populations and guilds in the Monte desert, Argentina. Journal of Field Ornithology 63:294-308.

Marone, L., López de Casande, J. \& Cueto, V.R. 1997. Patterns of habitat selection by wintering and breeding granivorous birds in the central Monte desert, Argentina. Revista Chilena de Historia Natural 70:73-81.

Marquet, P. 1994. Diversity of small mammals in the pacific coastal desert of Perú and Chile and in the adjacent andean area: Biogeography and community structure. Australian Journal of Zoology 42:527-542.

Marquet, P., Bozinovic, F., Bradshaw, G.A., Cornelius, C., González, H., Gutierres, J.R., Hajek, H.R., Lagos, J.A., López-Cortez, F., NúÑez, L., Rosello, E.F., Santoro, C., Samaniego, H., Standen, V.G., Torres-muRA, J.C. \& Jaksic, F.M. 1998. Los ecosistemas del desierto de Atacama y área andina adyacente en el norte de Chile. Revista Chilena de Historia Natural 71:593-617.

Meserve, P.L. \& Glanz, W.E. 1978. Geographical ecology of small mammals in the northern chilean arid zone. Journal of Biogeography 5:135-148.

MoRTON, S.R. 1985. Granivory in arid regions: comparison of Australia with North and South America. Ecology 66(6):1859-1866.

Morton, S.R. 1993. Determinants of diversity in animal communities of arid Australia. En: Species diversity in ecological communities (Eds. Ricklefs R.E. \& Schlutter, D.), pp. 159-169. University of Chicago Press. Chicago, USA.

Morton, S.R. \& Davies, P.H. 1983. Food of zebra finch (Peophila guttata), and an examination of granivory in birds of the Australian arid zone. Australian Journal of Ecology 8:235-243.

Noy-Meier, I. 1973. Desert ecosystems: environment and producers. Annual Review of Ecology and Systematics 4:25-51.

Rau, J., Zuleta, C., Gantz, A., Saiz, F., Cortez, A., Yates, L., Spotorno, A. \& Couve, E. 1998. Biodiversidad de artrópodos y vertebrados terrestres del norte grande de Chile. Revista Chilena de Historia Natural 71:527-554.

Ricklefs, R.E. 1987. Community diversity: Relative roles of local and regional processes. Science 235:167-171.

Rosenzweig, M.L. 1995. Species diversity in space and time. Cambridge University Press. 436 pp.

SChlutter, D. \& Ricklefs, R.E. 1993. Species diversity: An introduction to the problem. En: Species diversity in ecological communities (Eds. Ricklefs R.E. \& D. Schlutter), pp.1-10. The University of Chicago Press. USA.

Spotorno, A.E., Zuleta, C., Gantz, A., Saiz, F., Rau, J., Rosenmann, M., Cortéz, A., Yates, G., Couve, E. \& MARín, J.C. 1998. Sistemática y adaptación de mamíferos, aves e insectos fitófagos de la Región de Antofagasta, Chile. Revista Chilena de Historia Natural 71: 501-526.

Spotorno, A.E. \& Veloso, A. 1990. Flora y Fauna of the Altiplano. En: Strategies in Human Adaptations to a rigorous environment: the Aymara (Eds. Schull, W.J. \& Rothhamer, H.), pp. 19-32. Kluwer Academic Publications. Dordrecht, Netherland.

Tongway, D.J., Cortina, J. \& Maestre, F.T. 2004. Heterogeneidad espacial y gestión de medios semiáridos. Ecosistemas 13(1):2-15.

Vuilleumier, F. \& Simberloff, D. 1980. Ecology versus history as determinants of patchy and insular distributions in high Andean birds. Evolutionary Biology 12:235-379.

WhitTAKeR, R.H. 1972. Evolution and measurement of species diversity. Taxon 21:213-251.

WiENS, J.A. 1985. Vertebrate responses to environmental patchiness in arid and semiarid ecosystems. En: The ecology of natural disturbance and patch dynamics (Eds. Pickett, S.T.A. \& White, P.S.), pp: 169-193. Academic Press. New York, USA.

WIENS, J.A. 1991. Ecological similarity of shrub-desert avifauna of Australia and North America. Ecology 72:479-495. 